\title{
PHYTOPLANKTON SEASONAL DIVERSITY OF TADOBA LAKE (TATR) DISTRICT CHANDRAPUR MAHARASHTRA STATE, INDIA
}

\section{P.M.Telkhade ${ }^{1}$, R.V. Tijare $^{2}$ and S B Zade ${ }^{3}$}

1 Dept. of Zoology, Arts Commerce and Science College, Tukum, Chandrapur.(M.S) India.

${ }^{2}$ Dept. of Zoology, Institute of Science Nagpur (M.S) India.

2 P G T D Zoology, R T M Nagpur University, Nagpur, (M.S) India. Corresponding Author Email: ptelkhade@yahoo.in

\section{ABSTRACT:}

The present paper reports the seasonal phytoplankton diversity in Tadoba lake District, Chandrapur, Maharashtra state. Tadoba Andhari Tiger Reserve in the Chandrapur District in Eastern Maharashtra represents a unique habitat for Wildlife in Central India. The oldest National Park of the State. The present investigation was carried out to evaluate the seasonal population of planktonic algae of the Tadoba Lake during June 2005 - May 2006. The samples were collected during every month. The sample analysis showed great diversity in phytoplankton consisting 35 species belonging to four groups viz, Chlorophyceae, Bacillariophyceae, Cynophyceae and Euglenophyceae. Chlorophyceae were dominant by contributing 19 species followed by Cynophyceae by 08 species, Bacillariophyceae by 06 species and Euglenophyceae by 02 species. Seasonally, phytoplankton was dominant during winter season and least during monsoon.

Keywords: Tadoba lake, Phytoplankton, Diversity.

\section{INTRODUCTION:}

The phytoplankton plays their role as primary producers in an aquatic ecosystem. Their qualitative and quantitative estimation provide good indices of water quality and capacity of water to sustain heterotrophic communities. It is the base of most of the reservoir food webs and fish production is linked to phytoplankton production (Misra et.al., 1992). Phytoplankton forms the basic link of food chain in aquatic ecosystem. Plankton constitutes the vary basis of nutritional cycle of an ecosystem (Kaushik et al., 1991). They serve as food for fishes directly or indirectly. Some species have been associated with noxious blooms, sometimes creating of offensive tastes and odours or toxic conditions, planktonic organisms are predetermination in lentic environments. Moreover 
number and species of phytoplankton serves to determine the quality of water body and they make the water greenish. Phytoplankton of freshwater bodies has been studied extensively in India, Bilgrami, (1989), Verma, and Mohanty (1995), Kumar (1998) and Khanna and Yadav (2009). The present investigation was carried out to focus attention on the seasonal species population density of phytoplankton.

\section{MATERIAL AND METHODS:}

The samples were collected once in a month for a period of six month from June 2005 - May 2006 during 8.30 a.m. to 9.30 a.m. The samples were collected by filtering 50 liters of water through plankton net. The plankton mesh of size $56.00 \mu \mathrm{m}$ made up of bolting silk cloth. The samples were allowed to settle, centrifuged and the concentrate was made up to $50 \mathrm{ml}$ with preservation of $4 \%$ formalin. Preserved samples were examined under binocular microscope and identified using pertinent literature, Edmondson (1959) and Trivedi and Goel (1960).

\section{RESULT AND DISCUSSION:}

Phytoplankton also plays a vital role in the biological treatment of organic waste in the water, as during mineralization of organic matter by bacteria they supply the oxygen. Therefore evaluation of population, diversity, periodicity, succession, biomass and productivity of phytoplankton is very important in monitoring of water bodies. Phytoplankton is very much sensitive to the chemical parameters of water and any alteration in them leads to change in plankton communities in terms of tolerance, abundance, diversity and dominance in freshwater body largely depends on availability of nutrient such as nitrates, phosphates, silica etc. Production of phytoplankton greatly depends upon the availability of nutrients in general nitrogen and phosphate in particular (Syrett, 1981). In the present investigation, total 35 species belonging to four groups and phytoplankton diversity presented in Table -1.1 . 
and Photo plate 13. Chlorophyceae (19 species), Cynophyceae (08 species), Bacillariophyceae (06 species), and Euglenophyceae (02 species) among them maximum density was of Chlorophyceae followed by Cynophyceae, Bacillariophyceae and Euglenophyceae. Veerendra et al., (2006) identified 34 species of phytoplankton under four classes among them maximum density was of Bacillariophyceae followed by Chlorophyceae, Cynophyceae and Euglenophyceae in Mani reservoir, Karnataka.

In phytoplankton Chlorophyceae (202.25 \pm 117.6) ind/1tr shows dominance followed by Bacillariophyceae (167.33 \pm 132.03$)$ ind/1tr and Cynophyceae $(111.927 \pm 76.80)$ ind/ltr while least appearance was shown by Euglenophyceae $(59.00 \pm 35.53)$ ind/ltr during the year 2005-06. Seasonally phytoplankton was dominant during winter season and least density was during monsoon. In the present investigation maximum density of Chlorophyceae was observed during winter and minimum density during monsoon while moderate during summer season. The most dominant species Spirogyra spp., Zygnema spp., Ulothrix spp., Closterium spp. and least count was shown by Volvox spp., Oedogonium spp., Chlorella sp. and Pediastrum spp, Khanna and Bhutiani (2003) stated that Chlorophyceae was found to be dominating group at Sitapur pond, Hardwar. The phytoplankton diversity and density is controlled by water quality and other biotic communities in a water body (Reid and Wood, 1976).

Bacillariophyceae shows its dominance in winter season and least in summer and moderate in monsoon season. Bacillariophyceae was dominant during winter season and least in during monsoon season of 2005-06. Cynophyceae was maximum during summer and minimum in rainy season. George (1962) has observed that high temperature is the principal factor for growth of blue green algae. The diversity of Nostoc spp. was very less, it is an indication that lake is not polluted. Hutchinson (1967) observed minimum density in winter and maximum in warmer months. Chakraborthy et al. (1959) 
abundance of blue green algae is associated with high temperature, $\mathrm{pH}$ and low D.O., the present observation support the study.

Euglenophyceae shows its least count on all the sampling stations. Euglenophyceae was represented by only two genera namely Phacus spp. and Euglena spp. Euglenophyceae member was counted maximum during winter and moderate during monsoon and minimum in summer season. The similar study by Pawar et al., (2006) estimated that the density of Euglenophyceae was greater during winter season. Euglenophyceae shows the least appearance in phytoplankton during present investigation.

The study made on phytoplankton of water bodies all over the world has shown marked difference in the floristic composition with different levels of pollution which has prompted several workers to use algal composition as the indicator of the level of pollution (Rawson, 1956). Phytoplankton are the basic members in the aquatic ecosystem and hence changes in the phytoplankton population have a direct link with the change of water quality in any aquatic medium.

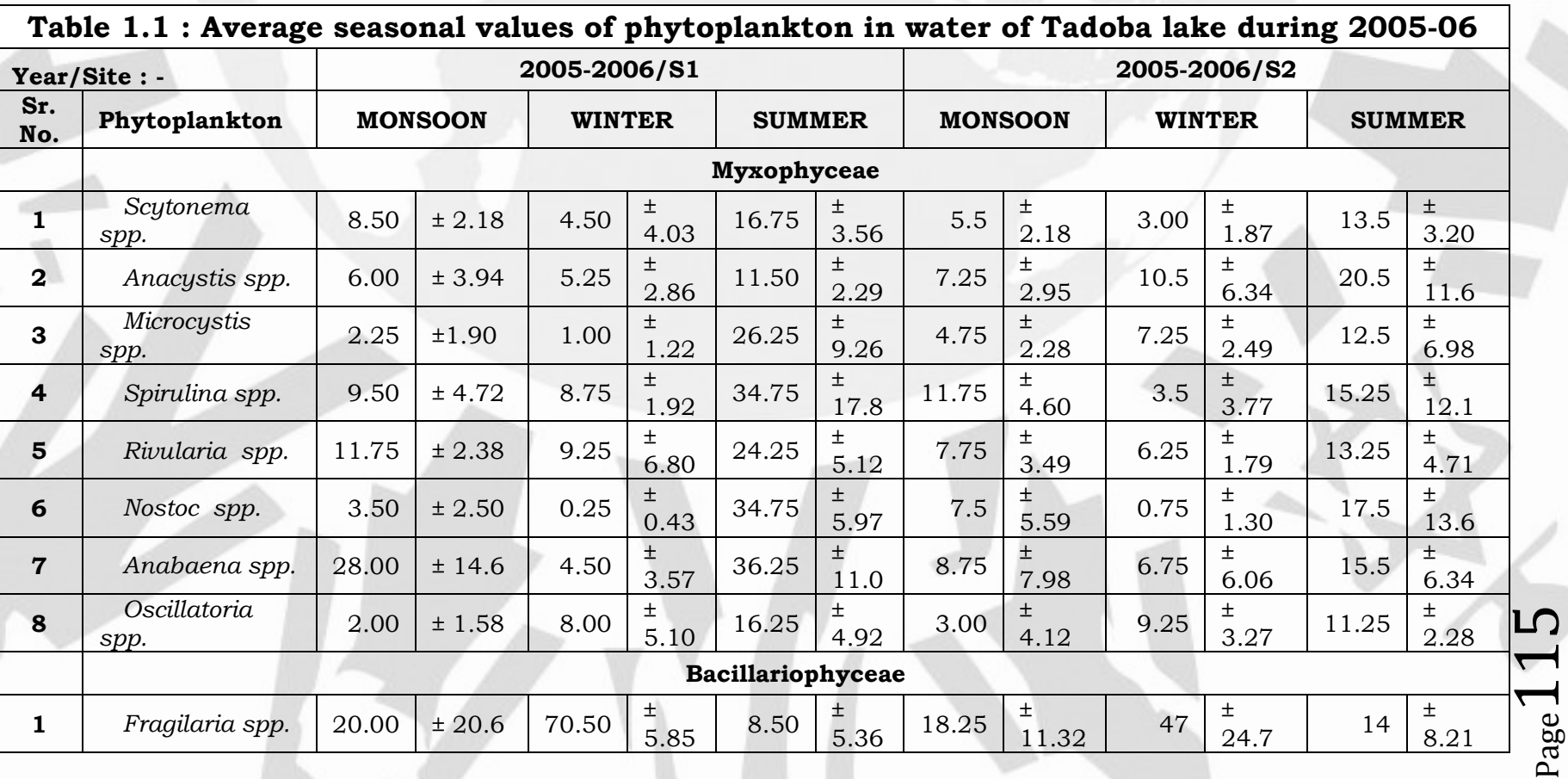


International Journal of Researches in Biosciences, Agriculture \& Technology
September 2013

Issue-1, Volume-1
ISSN No. (Online):

2347-517X

\begin{tabular}{|c|c|c|c|c|c|c|c|c|c|c|c|c|c|}
\hline 2 & Diatoma spp. & 30.25 & \pm 40.1 & 65.00 & $\begin{array}{l} \pm \\
32.0\end{array}$ & 40.75 & $\begin{array}{l} \pm \\
15.8\end{array}$ & 46.25 & $\begin{array}{l} \pm \\
30.16\end{array}$ & 110.3 & $\begin{array}{l} \pm \\
28.6\end{array}$ & 30.8 & $\begin{array}{l} \pm \\
11.6\end{array}$ \\
\hline 3 & Navicula spp. & 46.25 & \pm 35.8 & 55.50 & $\begin{array}{l} \pm \\
38.1\end{array}$ & 28.00 & $\begin{array}{l} \pm \\
17.8\end{array}$ & 62.5 & $\begin{array}{l} \pm \\
19.7 \\
\end{array}$ & 105.5 & $\begin{array}{l} \pm \\
21.5\end{array}$ & 15.5 & \pm 7.6 \\
\hline 4 & $\begin{array}{l}\text { Mastogloia } \\
\text { spp. }\end{array}$ & 52.00 & 3.24 & 48.00 & $\begin{array}{l} \pm \\
14.2 \\
\end{array}$ & 17.75 & $\begin{array}{l} \pm \\
13.2 \\
\end{array}$ & 2.75 & \pm 3.2 & 34.25 & $\begin{array}{l} \pm \\
18.9\end{array}$ & 12.75 & $\begin{array}{l} \pm \\
11.0\end{array}$ \\
\hline 5 & Pinnularia spp. & 5.00 & \pm 21.8 & 35.00 & $\begin{array}{l} \pm \\
25.8\end{array}$ & 22.50 & $\begin{array}{l} \pm \\
13.7\end{array}$ & 31.00 & \pm 6.7 & 104.3 & $\begin{array}{l} \pm \\
22.4 \\
\end{array}$ & 22.3 & $\begin{array}{l} \pm \\
10.2\end{array}$ \\
\hline \multirow[t]{2}{*}{6} & Nitzchia spp. & 0.00 & \pm 0.00 & 0.00 & $\begin{array}{l} \pm \\
0.00\end{array}$ & 0.00 & $\begin{array}{l} \pm \\
0.00\end{array}$ & 0.00 & \pm .00 & 0.00 & $\begin{array}{l} \pm \\
0.00\end{array}$ & 0.00 & $\begin{array}{l} \pm \\
0.00\end{array}$ \\
\hline & \multicolumn{13}{|c|}{ Chlorophyceae } \\
\hline 1 & Vaucheria spp. & 2.25 & \pm 1.48 & 18.00 & $\begin{array}{l} \pm \\
3.5 \\
\end{array}$ & 10.25 & $\begin{array}{l} \pm \\
5.80\end{array}$ & 2.5 & $\begin{array}{l} \pm \\
2.872 \\
\end{array}$ & 15.25 & $\begin{array}{l} \pm \\
5.540\end{array}$ & 1.75 & $\begin{array}{l} \pm \\
1.785 \\
\end{array}$ \\
\hline 2 & $\begin{array}{l}\text { Oedogonium } \\
\text { spp. }\end{array}$ & 12.75 & \pm 1.48 & 15.50 & $\begin{array}{l} \pm \\
1.50 \\
\end{array}$ & 6.50 & $\begin{array}{l} \pm \\
4.30 \\
\end{array}$ & 5.75 & \pm 5.4 & 16.25 & $\begin{array}{l} \pm \\
3.202 \\
\end{array}$ & 2.25 & $\begin{array}{l} \pm \\
2.487 \\
\end{array}$ \\
\hline 3 & Ulothrix spp. & 6.25 & \pm 5.31 & 33.25 & $\begin{array}{l} \pm \\
7.60\end{array}$ & 11.75 & $\begin{array}{l} \pm \\
7.01 \\
\end{array}$ & 5 & $\begin{array}{l} \pm \\
4.950 \\
\end{array}$ & 24.75 & $\begin{array}{l} \pm \\
10.4 \\
\end{array}$ & 5.25 & $\begin{array}{l} \pm \\
3.562 \\
\end{array}$ \\
\hline 4 & $\begin{array}{l}\text { Micrasterias } \\
\text { spp. }\end{array}$ & 10.75 & \pm 9.04 & 10.50 & $\begin{array}{l} \pm \\
2.06 \\
\end{array}$ & 0.25 & $\begin{array}{l} \pm \\
0.43\end{array}$ & 8.5 & $\begin{array}{l} \pm \\
6.874 \\
\end{array}$ & 27.75 & $\begin{array}{l} \pm \\
23.5 \\
\end{array}$ & 3.5 & $\begin{array}{l} \pm \\
4.387 \\
\end{array}$ \\
\hline 5 & Volvox spp. & 3.25 & \pm 2.86 & 1.00 & $\begin{array}{l} \pm \\
1.22 \\
\end{array}$ & 4.50 & $\begin{array}{l} \pm \\
2.96\end{array}$ & 1.25 & $\begin{array}{l} \pm \\
1.299\end{array}$ & 3 & $\begin{array}{l} \pm \\
2.739\end{array}$ & 4.25 & $\begin{array}{l} \pm \\
4.265\end{array}$ \\
\hline 6 & $\begin{array}{l}\text { Pediastrum } \\
\text { spp. }\end{array}$ & 3.50 & \pm 3.35 & 11.00 & $\begin{array}{l} \pm \\
4.95 \\
\end{array}$ & 0.00 & $\begin{array}{l} \pm \\
0.00\end{array}$ & 2.75 & $\begin{array}{l} \pm \\
1.479 \\
\end{array}$ & 15.25 & $\begin{array}{l} \pm \\
2.861 \\
\end{array}$ & 1.25 & $\begin{array}{l} \pm \\
1.299 \\
\end{array}$ \\
\hline 7 & Chlorella spp. & 3.75 & \pm 2.49 & 10.75 & $\begin{array}{l} \pm \\
3.70 \\
\end{array}$ & 2.75 & $\begin{array}{l} \pm \\
3.27 \\
\end{array}$ & 5.25 & $\begin{array}{l} \pm \\
2.278 \\
\end{array}$ & 14.5 & $\begin{array}{l} \pm \\
3.571 \\
\end{array}$ & 1.25 & $\begin{array}{l} \pm \\
1.299 \\
\end{array}$ \\
\hline 8 & Gleocystis spp. & 4.75 & \pm 5.54 & 15.75 & $\begin{array}{l} \pm \\
2.59\end{array}$ & 3.25 & $\begin{array}{l} \pm \\
5.63\end{array}$ & 0.75 & $\begin{array}{l} \pm \\
0.829\end{array}$ & 4.25 & $\begin{array}{l} \pm \\
2.861\end{array}$ & 0.75 & $\begin{array}{l} \pm \\
.829 \\
\end{array}$ \\
\hline 9 & $\begin{array}{l}\text { Cladophora } \\
\text { spp. }\end{array}$ & 2.00 & \pm 1.58 & 12.75 & $\begin{array}{l} \pm \\
4.32\end{array}$ & 4.00 & $\begin{array}{l} \pm \\
4.30\end{array}$ & 1.75 & $\begin{array}{l} \pm \\
1.479\end{array}$ & 9.75 & $\begin{array}{l} \pm \\
4.815\end{array}$ & 3.25 & $\begin{array}{l} \pm \\
2.586 \\
\end{array}$ \\
\hline 10 & $\begin{array}{l}\text { Cosmarium } \\
\text { spp. }\end{array}$ & 3.75 & \pm 3.34 & 13.50 & $\begin{array}{l} \pm \\
2.96\end{array}$ & 3.00 & $\begin{array}{l} \pm \\
5.20\end{array}$ & 3.25 & $\begin{array}{l} \pm \\
1.299\end{array}$ & 11.5 & $\begin{array}{l} \pm \\
1.803\end{array}$ & 1.5 & $\begin{array}{l} \pm \\
1.500 \\
\end{array}$ \\
\hline 11 & Spirogyra spp. & 15.75 & \pm 2.95 & 61.75 & $\begin{array}{l} \pm \\
11.6 \\
\end{array}$ & 13.25 & $\begin{array}{l} \pm \\
3.49 \\
\end{array}$ & 18.75 & $\begin{array}{l} \pm \\
4.085\end{array}$ & 42.25 & $\begin{array}{l} \pm \\
10.45\end{array}$ & 12.75 & $\begin{array}{l} \pm \\
4.380 \\
\end{array}$ \\
\hline 12 & Zygnema spp. & 17.75 & \pm 5.89 & 50.75 & $\begin{array}{l} \pm \\
10.6 \\
\end{array}$ & 15.50 & $\begin{array}{l} \pm \\
5.36\end{array}$ & 17 & $\begin{array}{l} \pm \\
15.2 \\
\end{array}$ & 34.5 & $\begin{array}{l} \pm \\
10.5\end{array}$ & 7.00 & $\begin{array}{l} \pm \\
6.442 \\
\end{array}$ \\
\hline 13 & $\begin{array}{l}\text { Closterium } \\
\text { spp. }\end{array}$ & 16.25 & \pm 4.71 & 32.00 & $\begin{array}{l} \pm \\
10.0\end{array}$ & 6.00 & $\begin{array}{l} \pm \\
4.74\end{array}$ & 3.75 & $\begin{array}{l} \pm \\
4.918\end{array}$ & 7.5 & $\begin{array}{l} \pm \\
8.201\end{array}$ & 4.25 & $\begin{array}{l} \pm \\
5.761\end{array}$ \\
\hline 14 & Ceratium spp. & 4.75 & \pm 4.49 & 14.50 & $\begin{array}{l} \pm \\
9.94\end{array}$ & 1.00 & $\begin{array}{l} \pm \\
1.73 \\
\end{array}$ & 1.75 & $\begin{array}{l} \pm \\
1.785\end{array}$ & 13.00 & $\begin{array}{l} \pm \\
4.743\end{array}$ & 2.5 & $\begin{array}{l} \pm \\
3.775\end{array}$ \\
\hline 15 & Pandorina spp. & 13.25 & \pm 8.44 & 22.00 & $\begin{array}{l} \pm \\
6.82 \\
\end{array}$ & 0.000 & $\begin{array}{l} \pm \\
0.00\end{array}$ & 0.00 & $\begin{array}{l} \pm \\
0.00 \\
\end{array}$ & 0.00 & $\begin{array}{l} \pm \\
0.00\end{array}$ & 0.00 & $\begin{array}{l} \pm \\
0.00 \\
\end{array}$ \\
\hline 16 & $\begin{array}{l}\text { Microspora } \\
\text { spp. }\end{array}$ & 4.75 & \pm 4.21 & 13.00 & $\begin{array}{l} \pm \\
7.45 \\
\end{array}$ & 0.00 & $\begin{array}{l} \pm \\
0.00\end{array}$ & 10.5 & $\begin{array}{l} \pm \\
5.172\end{array}$ & 35.25 & $\begin{array}{l} \pm \\
9.808\end{array}$ & 1.00 & $\begin{array}{l} \pm \\
1.732\end{array}$ \\
\hline 17 & Vorticella spp. & 0.00 & \pm 0.00 & 0.00 & $\begin{array}{l} \pm \\
\\
0.00\end{array}$ & 0.00 & $\begin{array}{l} \pm \\
0.00 \\
\end{array}$ & 0 & $\begin{array}{l} \pm \\
0.00 \\
\end{array}$ & 0 & $\begin{array}{l} \pm \\
0.00 \\
\end{array}$ & 0.00 & $\begin{array}{l} \pm \\
0.00 \\
\end{array}$ \\
\hline 18 & $\begin{array}{l}\text { Coelastrum } \\
\text { spp. }\end{array}$ & 1.75 & \pm 1.48 & 1.50 & $\begin{array}{l} \pm \\
1.12 \\
\end{array}$ & 1.00 & $\begin{array}{l} \pm \\
1.73 \\
\end{array}$ & 1.75 & $\begin{array}{l} \pm \\
0.829 \\
\end{array}$ & 2.25 & $\begin{array}{l} \pm \\
1.090\end{array}$ & 0.00 & $\begin{array}{l} \pm \\
0.00 \\
\end{array}$ \\
\hline 19 & $\begin{array}{l}\text { Chlorocloster } \\
\text { spp. }\end{array}$ & 0.00 & \pm 0.00 & 0.00 & $\begin{array}{l} \pm \\
0.00\end{array}$ & 0.00 & $\begin{array}{l} \pm \\
0.00 \\
\end{array}$ & 0.00 & $\begin{array}{l} \pm \\
0.00 \\
\end{array}$ & 0.00 & $\begin{array}{l} \pm \\
0.00\end{array}$ & 0.00 & $\begin{array}{l} \pm \\
0.00\end{array}$ \\
\hline & & & & & & lenop & yceae & & & & & & \\
\hline 1 & Phacus spp. & 21.25 & \pm 5.21 & 19.75 & $\begin{array}{l} \pm \\
10.5 \\
\end{array}$ & 8.75 & $\begin{array}{l} \pm \\
5.99 \\
\end{array}$ & 20 & $\begin{array}{l} \pm \\
6.403 \\
\end{array}$ & 25.25 & $\begin{array}{l} \pm \\
8.227\end{array}$ & 25 & $\begin{array}{l} \pm \\
11.24 \\
\end{array}$ \\
\hline 2 & Euglena spp. & 19.50 & \pm 1.802 & 20.50 & $\begin{array}{l} \pm \\
7.36\end{array}$ & 10.75 & $\begin{array}{l} \pm \\
7.46\end{array}$ & 13.25 & $\begin{array}{l} \pm \\
6.339 \\
\end{array}$ & 40 & $\begin{array}{l} \pm \\
31.78\end{array}$ & 9.75 & $\begin{array}{l} \pm \\
3.767\end{array}$ \\
\hline
\end{tabular}

Table 1.2 : Average seasonal values of phytoplankton in water of Tadoba lake during 2005-06 Year/Site : - $2005-2006 /$ S3

\begin{tabular}{c|l|l|l|l|l|l|l|}
$\begin{array}{c}\text { Sr. } \\
\text { No. }\end{array}$ & Phytoplankton & MONSOON & WINTER & SUMMER & MONSOON & WINTER & SUMMER \\
\hline
\end{tabular}




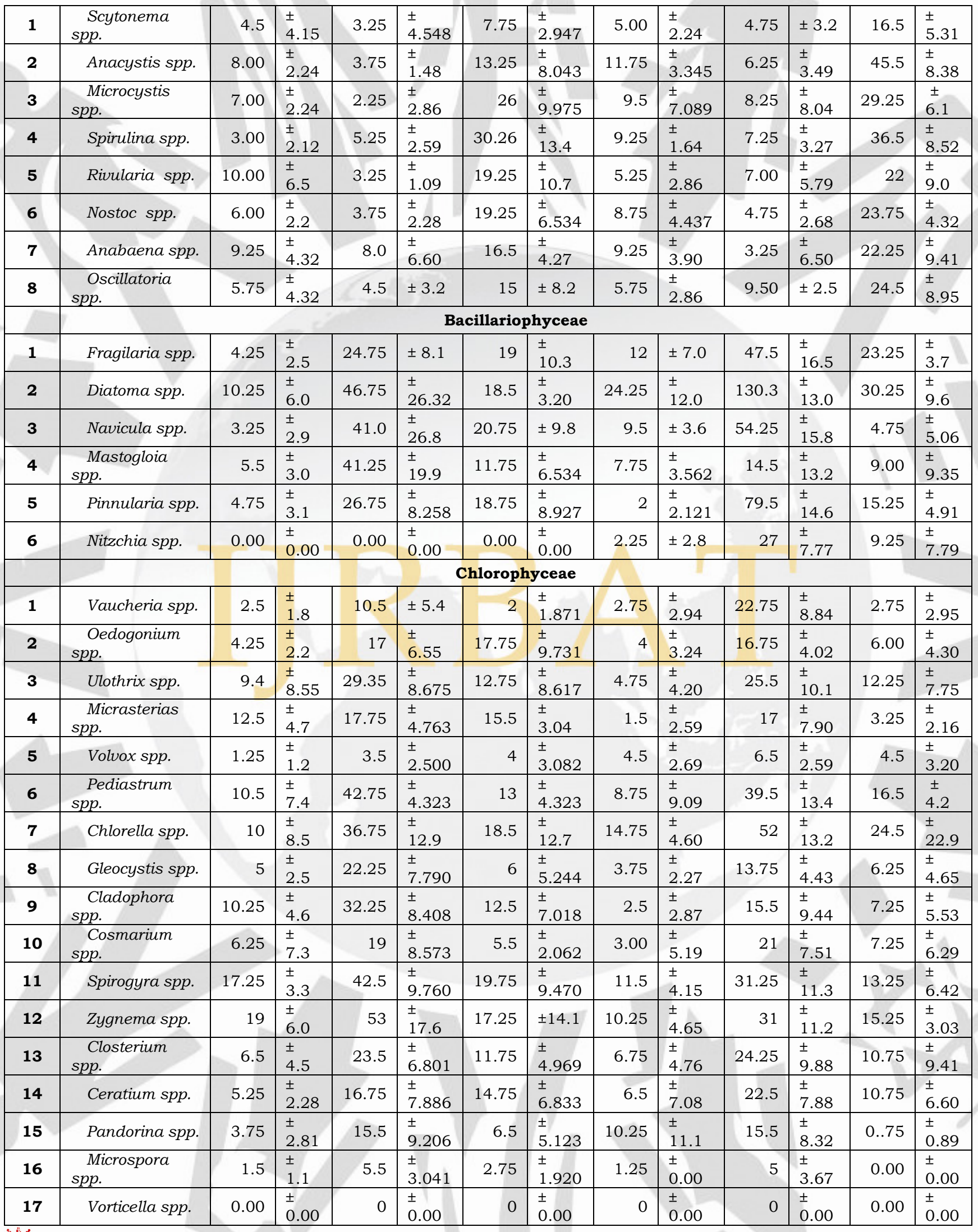


International Journal of Researches in Biosciences, Agriculture \& Technology

September 2013

Issue-1, Volume-1
ISSN No. (Online): 2347-517X

\begin{tabular}{|c|c|c|c|c|c|c|c|c|c|c|c|c|c|}
\hline 18 & $\begin{array}{l}\text { Coelastrum } \\
\text { spp. }\end{array}$ & 1.5 & $\begin{array}{l} \pm \\
1.6 \\
\end{array}$ & 6.75 & $\begin{array}{l} \pm \\
3.345 \\
\end{array}$ & 2.25 & $\begin{array}{l} \pm \\
2.278\end{array}$ & 0.75 & $\begin{array}{l} \pm \\
0.82\end{array}$ & 6.75 & $\begin{array}{l} \pm \\
3.56\end{array}$ & 1.5 & $\begin{array}{l} \pm \\
2.59\end{array}$ \\
\hline 19 & $\begin{array}{l}\text { Chlorocloster } \\
\text { spp. }\end{array}$ & 0.00 & $\begin{array}{l} \pm \\
0.00\end{array}$ & 0.00 & $\begin{array}{l} \pm \\
0.00\end{array}$ & 0.00 & $\begin{array}{l} \pm \\
0.00\end{array}$ & 0.00 & $\begin{array}{l} \pm \\
0.00\end{array}$ & 0.00 & $\begin{array}{l} \pm \\
0.00\end{array}$ & 0.00 & $\begin{array}{l} \pm \\
0.00\end{array}$ \\
\hline & \multicolumn{13}{|c|}{ Euglenophyceae } \\
\hline 1 & Phacus spp. & 9.75 & $\begin{array}{l} \pm \\
2.16\end{array}$ & 64.75 & $\begin{array}{l} \pm \\
23.2\end{array}$ & 25.25 & \pm .16 & 19 & $\begin{array}{l} \pm \\
5.477\end{array}$ & 64.25 & $\begin{array}{l} \pm \\
25.5\end{array}$ & 24.0 & $\begin{array}{l} \pm \\
12.4\end{array}$ \\
\hline 2 & Euglena spp. & 11.0 & $\begin{array}{l} \pm \\
6.2\end{array}$ & 49.25 & $\begin{array}{l} \pm \\
25.8\end{array}$ & 21.25 & $\begin{array}{l} \pm \\
10.20\end{array}$ & 15.75 & $\begin{array}{l} \pm \\
8.043\end{array}$ & 45.0 & $\begin{array}{l} \pm \\
27.3\end{array}$ & 9.00 & $\begin{array}{c} \pm \\
3.2\end{array}$ \\
\hline
\end{tabular}

\section{REFERENCES:}

Bilgrami, S. (1989): Bio-Monitoring of water quality of the ganga. In the Ganga project directorate Eds: Krishna Murthy, C. R Bilgrmi, K S Das, I. M. and Mathor, RP Northern Book Center, New Delhi: pp. 101 -106.

Chakraborty, R, D.P. Roy and S.B. Singh (1959): A qualitative study of the plankton and Physico-chemical conditions of the river Jamuna at Allahabad in 1954-1955. Indian J. fish. 6(1): pp.-186-203.

Edmondson, (1959): Freshwater Biology. Edn. Ward and Whippl, 2nd Edn. Johna Wiley sons Inc. New York: pp. 95-189.

Hutchinson, G.E. (1967): A treatise on limnology vo. II. Introduction to lake biology and the limnoplankton. John Wiley and Sons, N.Y. 115.

Kaushik, S., Agarkar, M.S. and Saxena, D.N. (1991): Water quality and periodicity of phytoplanktonic algae in Chambal tank, Gwalior, Madhya Pradesh. Bionature. (11): pp.87 - 94.

Khanna, D.R. and Bhutiani R. (2003): Ecological status of Sitapur pond at Hardwar (Uttaranchal), India. Indian J. Environ and Ecoplan.72 (1):pp. $175-178$.

Khanna, Vibha and Yadav, Indu (2009): Planktonic diversity in the holy lake of Pushkar, Ajmer Int. Jour Nature Environment and pollution Technology 8(2): pp. 339-342.

Kumar, S.(1998): Limnology of Kanjwan with reference to Plankton and macrophytes.M.Phil Disser. Univ.Jammu, India. 
Misra, S.R., Sharma, Sanjay and Yadav, R.K. (1992): Phytoplanktonic community in relation to environmental conditions of lentic water of Gwalior (M.P.) India. J. Env. Biol. 13 (4): pp. 291 - 296.

Pawar,S.K. Pulle, J.S. and Shende, K.M. (2006):The study on phytoplankton of Pethwadaj Dam Taluka Kandhar, District Nanded, Mahasrashatra J.Aqua .Biol., 21 (1): pp.6-7.

Rawson, D.S. (1956): Algal indicators of trophic lake types: Lmnol. Oceancgr. (1): pp.18- 25 .

Reid, G.K and Wood R.D. (1976): Ecology of inland waters and esturies D.Van Norstand Co.New York: pp. 485.

Syrett, P.T. (1981): Nitrogen metabolism in micro algae in physiological basis of phytoplankton ecology, Platt. T. (Ed.) Bull. Fish. Aquat. Sci. 210: pp.182210

Trivedi, R.K. and Goel, P.K.(1960):Chemical and Biological methods of water pollution studies. Environmental Publication. Karad.

Veerendra, D.N., Manjappa, S. and Puttaiah, E.T. (2006): Diversity of phytoplankton in Mani reservoir, Hosanagar, Karnataka. Indian J. Environ and Ecoplan.12 (2): pp.335-338.

Verma, J.P. and Mohanty, R.C.(1995): Phytoplankton of Malyanta pond of Laxmisagar and its correlation with physico-chemical parameters. Enviromedia Pollution Research. (14):pp.243-253. 
International Journal of Researches in Biosciences, Agriculture \& Technology
September 2013

Issue-1, Volume-1
ISSN No. (Online):

2347-517X

PHOTO PLATE NO. 13

\section{PHYTOPLANKTONS}

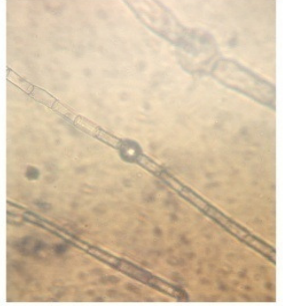

Oedogonium $s p$

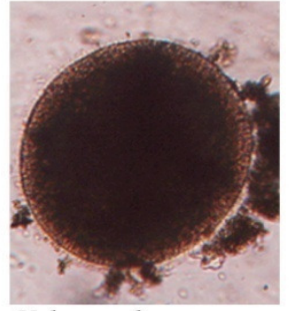

Volvox colony sp.

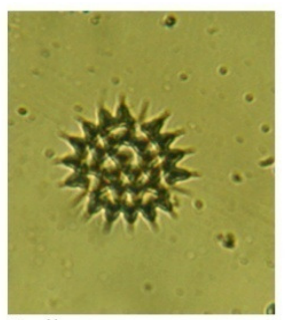

Pediastrum sp.

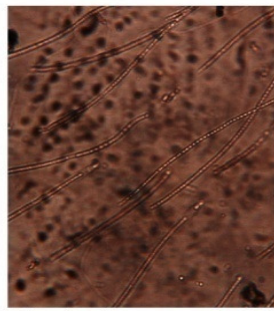

Rivularia $s p$.

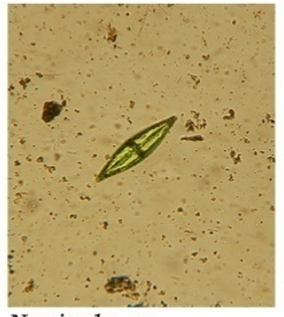

Navicula $s p$.

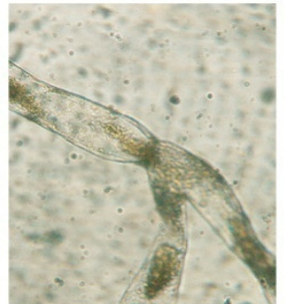

Voucheria sp.

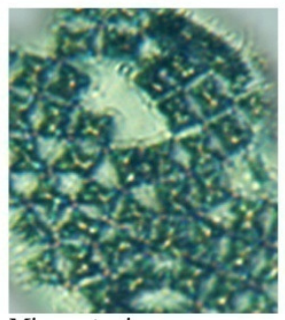

Micrasterius sp.

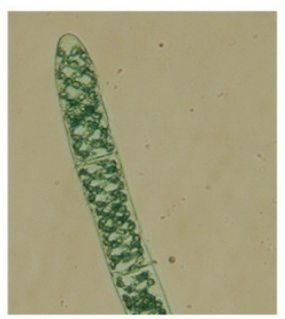

Spirogyra $s p$.

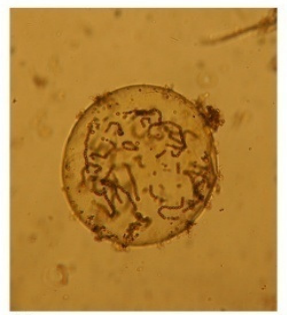

Nostoc sp.

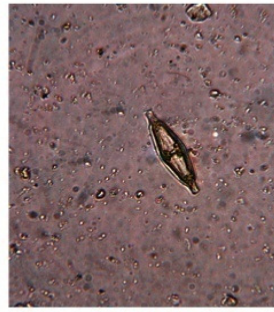

Nitzchia sp.

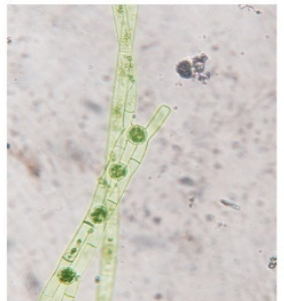

Zygnema sp.

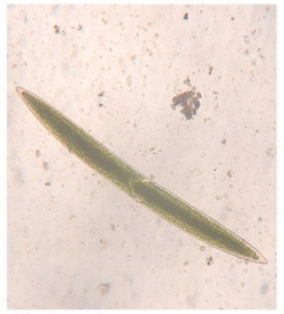

Coelastrum sp.

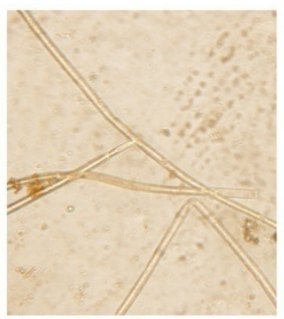

Scytonema sp.

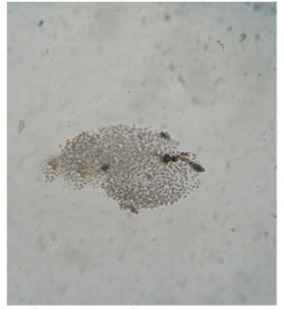

Microsystis $s p$.

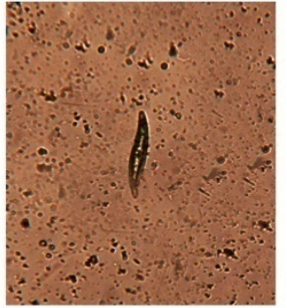

Gyrosigma sp.

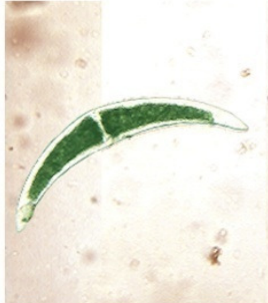

Closterium $s p$.

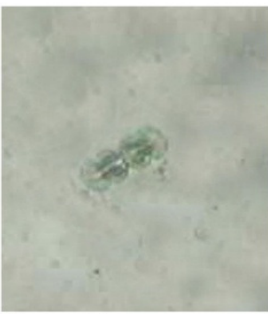

Cosmarium $s p$.

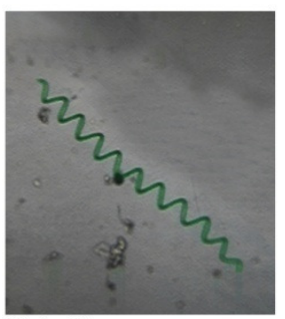

Spirulina $s p$.

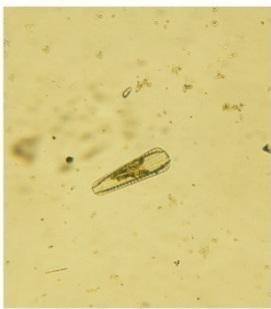

Fragilaria $s p$.

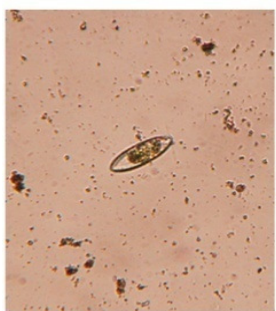

Diatoma sp.
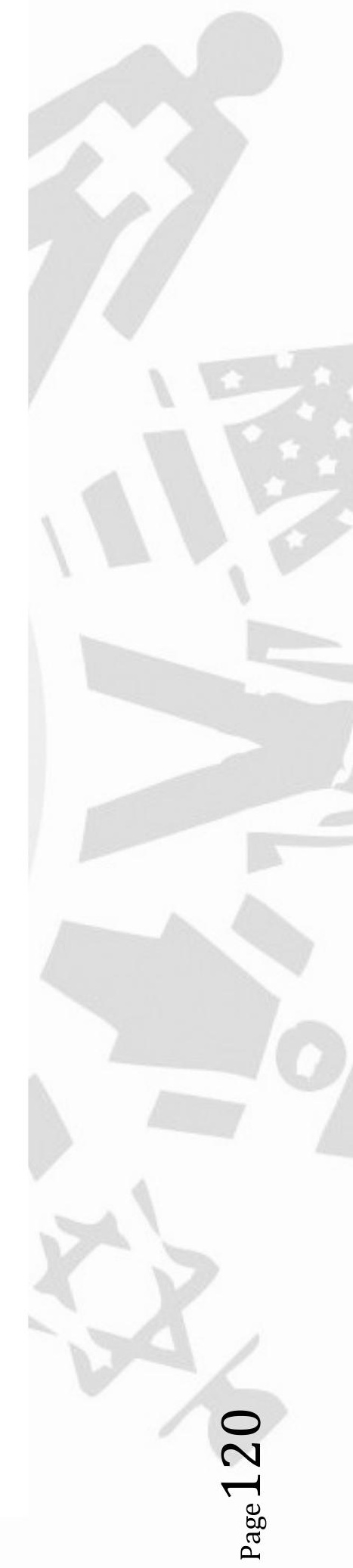